\title{
A Survey on Leach Protocol and its Enhanced Version
}

\author{
Inam Mohammad \\ M.Tech (C.S) Scholar \\ Graphic Era Hill University, Bhimtal, India
}

\begin{abstract}
Improvement in communications and computation Technology has enabled the development of low-cost, lowpower, small-size, and multifunctional sensor nodes in a wireless sensor network. The radio transmission and reception consumes a lot of energy, one of the important issues in wireless sensor network is the inherent limited battery power within network sensor nodes. So that battery power is a very important parameter in the algorithm design to increase lifetime of sensor nodes in the network. To maximizing the lifetime of sensor nodes, it is more suitable to divide the energy dissipated throughout the wireless sensor network in order to maximize overall network performance. Much research has been done in recent years, investigating different aspects like, low power protocols, network establishments, routing protocol, and coverage problems of wireless sensor networks. There are various routing protocols like location-aided, multi-path, data-centric, mobility-based, QoS based, heterogeneity-based, hierarchical routing, hybrid routing, etc., in which optimal routing can be achieved in the context of energy. In this paper, we focus only on hierarchical routing protocol and various LEACH-Based hierarchal routing protocols like LEACH-C, M-LEACH, E-LEACH, VLEACH etc. Most of the research in energy efficient data gathering in data centric applications of wireless sensor networks is motivated by LEACH
\end{abstract}

\section{Keywords}

WSN (Wireless Sensor Network), CHs (Clusters Head)

\section{INTRODUCTION}

Wireless Sensor Network is a network with many sensor devices (or sensor nodes) working together in a certain area. The sensor devices can form a multi-hop self-organized, ad hoc network using wireless communication to acquire and process information from objects, and then transmit it to the observer. The sensors, transceiver and observer are three key elements in a WSN. Due to the characteristics, WSNs have many applications such as industrial sensing, environmental detecting, earthquake predicting and military monitoring etc.

In recent years, many routing protocols for WSNs have been proposed [1-6] which can be classified into four classes: clustering-based routing protocols, data centric routing protocols, geographic-based routing protocol and hybrid routing protocol. A cluster based routing protocol grouped sensor nodes where each group of nodes has a cluster-head $(\mathrm{CH})$ or a gateway. Many clustering-based routing protocols have been proposed such as LEACH[3], LEACHC[4], HEED[5], TEEN[6] etc. Among them, LEACH is the most popular hierarchical routing algorithm for sensor networks.

Although band utilization can be also an important issue in wireless communication, however in a case of WSN, the first target is using energy with very high efficiency. The reason is that the battery on a sensor device cannot be replaced or charged. Therefore, the issue, how to make a network consume energy more efficiently, has become a very important research area.

(i) Any new technology that is in development phase gives a lot of opportunities to the young researchers and scientist to find some new ideas that help in development.

(ii) Balancing the power constraint is not an easy task. So it is the biggest task for researchers and scientists to improve it.

(iii) Power is a basic need for every sensor node or it is the backbone to every wireless sensor network.

(iv) All the critical applications that use wireless sensor networks have to deal with the network life because of limited power source.

\section{HIERARCHICAL ROUTING PROTOCOLS}

In hierarchical routing, the network is divided into clusters to achieve efficiency. The selection of cluster heads and formation of clusters are the two important criteria of hierarchical routing protocols. The advantage of hierarchical routing is data aggregation. The data from all member nodes are sent to the cluster head and then the cluster head forwards these data towards the sink after applying compression techniques. The aggregated data are easy to handle and simple to process. One of the major drawbacks of hierarchical routing is the increase in energy consumption of cluster heads due to their additional functions. The nodes are selected as cluster heads in rotation manner, which overcomes this issue. The selection of cluster heads and the formation of clusters in each round require more computations, which also causes more energy consumption.

LEACH [3] protocol is the basic clustering-based energyefficient routing protocol. The clustering techniques proved to be very useful to reduce the energy consumption and increase the network lifetime. The entire network is divided into clusters in the LEACH routing protocol. One sensor node in each cluster must act as a cluster head and all remaining sensor nodes are member nodes of that cluster. Communication between the member nodes and sink is only possible via the cluster head. From each cluster, only the cluster head can directly communicate with the sink. The cluster heads collect, aggregate, and forward the data from member nodes to the sink. The cluster head consumes more energy due to the additional functions and this node can die quickly if it continuously plays the role of a cluster head. LEACH resolved this problem by changing dynamically the role of nodes as cluster heads.

LEACH works in rounds. The operations that are carried out in each round consist of two phases known as setup and steady state phases. The organization of clusters and selection 
of cluster heads $(\mathrm{CHs})$ are done in the setup phase of the LEACH. The data are sent to the Sink during the second or steady state phase.

In the setup phase, the formation of clusters and the election process of cluster heads are performed. First of all, the whole network is divided into clusters. Now the cluster head election process starts in each cluster. There are many ways to elect the $\mathrm{CH}$. Some of the wireless nodes in the network ignore the negotiation process with other nodes and elect themselves autonomously as $\mathrm{CHs}$. The $\mathrm{CH}$ selection criteria of a member node are the recommended percentage $p$ and the earlier record as a $\mathrm{CH}$. If a node is not a $\mathrm{CH}$ in preceding $1 / \mathrm{P}$ rounds, it produces a number between zero and one (0-1). Only nodes with a generated number less than threshold $\mathrm{T}$ (n) are eligible to become $\mathrm{CHs}$. The formula used to calculate the value of threshold is given in equation 1 .

$$
\begin{aligned}
& T(n)=\left\{\frac{\mathrm{p}}{1-\mathrm{p}^{*}(\bmod 1 / \mathrm{p})} \quad \text { if } n \in \mathrm{G} \quad\right. \text { equationl } \\
& 0 \text { otherwise }
\end{aligned}
$$

Where

$\mathrm{G}=$ Group of nodes not selected as $\mathrm{CHs}$ in preceding $1 / \mathrm{p}$ rounds;

$\mathrm{p}=$ Recommended percentage of $\mathrm{CH}$;

$\mathrm{r}=$ Current round.

A node cannot be selected as a $\mathrm{CH}$ if it has already performed a $\mathrm{CH}$ role in the last $1 / \mathrm{p}$ rounds but all nodes that were $\mathrm{CHs}$ before $1 / \mathrm{p}$ rounds will again be candidates for the selection of $\mathrm{CHs}$ [3]. The uniform service of each node as a $\mathrm{CH}$ prevents the uneven energy consumption of the member nodes. The CSMA/CA protocol is used by the $\mathrm{CH}$ to broadcast its status. After receiving the broadcast messages from $\mathrm{CHs}$, the non-cluster head nodes use the Received Signal Strength Indication (RSSI) as a parameter to select their $\mathrm{CH}$. Each $\mathrm{CH}$ creates a Time Division Multiple Access (TDMA) schedule for their cluster members. The $\mathrm{CH}$ and member nodes communicate with each other during their assigned time slots in the steady state phase. The member node is only in active mode during its communication with a $\mathrm{CH}$. Otherwise the member node goes to sleep mode during an unallocated time slot. The management of the member node in this way reduces the energy consumption and increases the battery life of the node. The $\mathrm{CH}$ collects the data from all the cluster member nodes. The $\mathrm{CH}$ transmits that data to the base station after compression. The time duration of setup phase is lower than the steady state phase. Many protocols were proposed for the enhancement of LEACH. Some of them are discussed below.

The key features of LEACH are: (i) randomized rotation of the $\mathrm{CH}$ and corresponding clusters, (ii) local compression to reduce global communication, (iii) and localized coordination and control for cluster set-up and operation.

\section{Advantages of LEACH [7]}

(i) Each node has an equal chance to become a cluster head but cannot be selected as cluster head in a subsequent round so the load is shared between nodes.

(ii) Because LEACH uses Time Division Multiple Access (TDMA), it keeps cluster heads from unnecessary collisions.

(iii) LEACH can avoid a lot of energy dissipation by opening and closing member's communication interfaces in conformity with their allocated time slots.

\section{Limitations of LEACH [7]}

(i) Because LEACH uses single-hop communication, it cannot be deployed in networks spread over large distances.

(ii) Because cluster heads are elected only on the basis of probability, not taking energy into consideration,

(iii) LEACH cannot provide actual load balancing. Because cluster heads are elected on the basis of probability, uniform distribution cannot be ensured. So, there is a chance that the elected cluster heads are concentrated in one part of the network and some nodes might not have any cluster heads in their vicinity.

(iv) The idea of dynamic clustering brings extra overhead.

Problem in Direct Transmission and Multi-hop to overcome LEACH is proposed

* Problem Statement: The problem of high consumption of Energy during transmission remains in direct transmission. Utilization of power of a sensor node is major challenge for researchers to optimize node's life.

* Solution: Low Energy Adaptive Clustering Hierarchy $(\mathrm{LEACH})$ is a clustering-based protocol that utilizes randomized rotation of the cluster-heads to evenly distribute the energy load among the sensor nodes in the network

* Limitation: LEACH would not be able to cover large geographical areas of some square miles or more, because of the battery powered sensors there battery die very quickly the cluster head is miles away from the base station so it require more amout of energy for cluster head to send data to base station

\section{IMPROVED VERSIONS OF LEACH}

3.1 LEACH-C [4] is proposed by Heinzelman et al. The conventional LEACH protocol does not guarantee the best possible number of $\mathrm{CHs}$ and their effectual locations [3]. The problem is due to the clusters formation method used by the LEACH algorithm. LEACH-C is therefore proposed to enhance the cluster creation part of the LEACH protocol.

All nodes are required to send their ID, location, and energy information to the base station during the setup phase of LEACH-C [4]. The base station is responsible for assigning the role of $\mathrm{CH}$ to any member node by using its central control algorithm. The central control algorithm first specifies the average energy level and then compares that energy level to the energy level of the received signal energy [11]. The base station picks the optimal number of $\mathrm{CHs}$ from the nodes with an energy level greater than the average energy level. A list of IDs of these selected nodes is transmitted by the base station to all nodes. From this list, a node having minimum distance from its member nodes is elected as $\mathrm{CH}$ of that cluster. The approach used in LEACH-C reduces the energy consumption of $\mathrm{CH}$ and member nodes. The following assumptions are taken in this protocol:

(i) Every node can calculate its energy.

(ii) Every node knows its location.

(iii) Every node can communicate to the base station.

The successful data transmission during the steady state phase of LEACH-C increases. 
Some of the disadvantages of using the LEACH-C protocols are given below.

(i) During the setup phase, all the nodes in the network are required to send their information to the base station. This process causes additional energy consumption from each node.

(ii) The central control algorithm runs on the base station to select the CHs. The IDs of selected $\mathrm{CHs}$ are passed to all nodes. Every node needs to compare its ID with the IDs of CHs to determine its role as a $\mathrm{CH}$. These additional computations consume more energy from the nodes.

Some problem and solution of LEACH protocol.

* Problem Statement: The conventional LEACH protocol does not guarantee the best possible number of $\mathrm{CHs}$ and their effectual locations. The problem is due to the clusters formation method used by the LEACH algorithm.

* Solution: LEACH-C is therefore proposed to enhance the cluster creation part of the LEACH protocol. All nodes are required to send their ID, location, and energy information to the base station during the setup phase of LEACH-C

* Limitation: During the setup phase, all the nodes in the network are required to send their information to the base station. This process causes additional energy consumption from each node.

\subsection{LEACH-A (Advanced LEACH)}

In original $\mathrm{LEACH}$ protocol $\mathrm{CH}$ is responsible for transmits data directly to base station and cluster head $(\mathrm{CH})$ consume extra energy than cluster member (CM) nodes in network. ALEACH is extension of LEACH. Hence in Advanced LEACH protocol energy conserving and reliable data transfer both are improved [8]. In Advanced-LEACH, the data is processed with mobile agent technique.

Advanced-LEACH is proposed for the purpose of reliable data transfer, energy saving, decreasing the chance of failure of sensor nodes and for increasing the time interval before the first node dies. A-LEACH uses synchronized clock through which sensors get starting time of each round [8,9]. CAG nodes are set with time more energy than other nodes. CAG used as gateway or cluster head and rest nodes are used as normal nodes.

A-LEACH protocol has following advantages:

(i) The data is collaborates to reducing the quantity of data that is broadcasted to the Base Station [10].

(ii) The CAG nodes continue to send data to base station when all normal nodes die.

(iii) The Gateways extend the lifetime of network by reducing energy consumption.

(iv) Gateways reduce probability of node's failure and increase time interval before first node dies.

(v) Advanced-LEACH uses TDMA/CDMA techniques that create clustering on several levels. It saves more energy.

\subsection{LEACH-L (Energy Balanced LEACH)}

LEACH-L is energy balanced advanced multi-hop cluster based routing protocol. It is only depends upon distance. In LEACH-L the optimal hop counts are assumed. When cluster heads are sited near to BS (Base Station), then they directly communicate with BS. Cluster heads communicate via multi- hop mode when they are sited far away from the BS, and shortest communication distance is limited. In LEACH-L sensors nodes communicate with BS at different frequencies. The clusters are reorganized in each round and there are two phase: set-up phase and steady phase in each round. The new $\mathrm{CHs}$ are selected in each round. Load is circulated and balanced between all the nodes in the senor network. LEACHL covers large area than Mobile-LEACH because LEACH-L allocates equal power among all the senor nodes in the preperiod [8], [12].

\subsection{LEACH-B (Balanced LEACH)}

Balanced-LEACH is an improvement of original LEACH Protocol. In LEACH-B decentralized algorithms used for formation of clusters. In Leach-B sensor nodes have knowledge about its own position and destination receiver position and have no information about location of other sensor nodes. In Balanced-LEACH [13] data transmits using multiple access techniques. Each sensor nodes selects its cluster head, by analyzing the energy lost in the pathway between final recipient and itself. Efficiency of Balanced$L E A C H$ protocol is better as compared to original LEACH protocol.

\subsection{K-LEACH [14]}

Uses the K-medoids clustering algorithm to obtain highly uniform clustering of nodes and very good choices of cluster heads and it is a very well known fact that energy retention of a WSN is highly dependent on the grouping or clustering of transmitting and receiving nodes. In LEACH protocol, however, cluster formation is random and this may lead to non-uniform cluster sizes as well as poor formation of clusters. Some clusters may have more nodes and some may have very few nodes.

K-LEACH [14] considers least distant from the center of cluster as a criterion for a node to be chosen as a cluster head $(\mathrm{CH})$ during cluster head selection procedure (from second round onwards), whereas LEACH protocol does random selection of $\mathrm{CHs}$, this again may lead to poor to very poor selection of $\mathrm{CHs}$ which will consequently lead to highly inefficient energy retention by the network.

\subsection{K-LEACH [14]}

Protocol improves the clustering and cluster head selection procedure. For the first round of communication, in setup phase we use the K-medoids algorithm for cluster formation, which ensures uniform clustering. The cluster formation by $\mathrm{K}$-medoids algorithm ensures best clustering and selection of cluster head using Euclidian distance at the nearer or at the center of cluster always gives most energy efficient solution in WSN. From second round onwards cluster heads are selected based on the next nearest node to the first round cluster head and so on. We have applied clustering till the smallest cluster nodes are not considered. Then we have applied MRE till we get unique cluster heads, but as soon as we get duplicate cluster heads due to dynamic clustering we switch to random selection of cluster head nodes from amongst the alive nodes. K-LEACH is divided into many rounds, and each round contains cluster formation phase and Steady state phase.

\section{Cluster formation phase:}

(i) For the first round clusters are formed using Kmedoids cluster formation algorithm and cluster heads are selected as a node which lies at the center or nearer to the center of cluster using Euclidian distance. For rest of the rounds nodes nearest to the 
cluster head of the first round selection is chosen as cluster head.

(ii) Some nodes that turn into cluster heads as per above conditions send their cluster head announcement information to inform other nodes. The other nodes turn up as non cluster head nodes send cluster joining information to cluster head.

(iii) Cluster heads prepare their TDMA schedule.

\section{Steady state phase:}

(i) Nodes in a cluster, sends their data according to TDMA schedule, and cluster head receives, and aggregates the data.

(ii) The cluster heads will send their data directly to the base station.

This way the limitation of random clustering of LEACH protocol is addressed by uniform clustering to balance the load of entire network among all the nodes.

\subsection{V-LEACH [15]}

New version of LEACH protocol, the cluster contains; $\mathrm{CH}$ (responsible only for sending data that is received from the cluster members to the $\mathrm{BS}$ ), vice- $\mathrm{CH}$ (the node that will become a $\mathrm{CH}$ of the cluster in case of $\mathrm{CH}$ dies), cluster nodes (gathering data from environment and send it to the $\mathrm{CH}$ ).

In the original $\mathrm{LEACH}$, the $\mathrm{CH}$ is always on receiving data from cluster members, these data and then sends it to the BS that might be located far away from it. The $\mathrm{CH}$ will die earlier than the other nodes in the cluster because of its operation of receiving, sending and overhearing. When the $\mathrm{CH}$ die, the cluster will become useless because the data gathered by cluster nodes will never reach the base station. In V-LEACH protocol, besides having a $\mathrm{CH}$ in the cluster, there is a vice- $\mathrm{CH}$ that takes the role of the $\mathrm{CH}$ when the $\mathrm{CH}$ dies because the reasons we mentioned above by doing this, cluster nodes data will always reach the BS; no need to elect a new $\mathrm{CH}$ each time the $\mathrm{CH}$ dies. This will extend the overall network life time. The main problem with $\mathrm{LEACH}$ protocol lies in the random selection of cluster heads. There exists a probability that the cluster heads formed are unbalanced and may remain in one part of the network making some part of the network unreachable. This problem is resolved by using the concept of V-Leach [15]. V-Leach uses the concept of alternate Cluster Head called Vice Cluster Head. As a Cluster Head dies it is replaced by the Vice Cluster Head. But in case of Vice Cluster Head Dies, it does not provide solution for that and the network start reducing the energy very fast and finally the network dies completely. Now in V-leach The decision of the Cluster head and Vice Cluster head selection is on the basis of Energy, Distance and Residual Energy. The V-leach will improve the network life and total communication over the network.

\subsection{M-LEACH}

In LEACH protocol the information is transmitted from cluster head $(\mathrm{CH})$ to base station (BS) node through single hop communication no matter the distance between BS and $\mathrm{CH}$. Energy consumption will be more if distance is far. This M-LEACH protocol modifies LEACH allowing sensor nodes to use multi-hop communication within the cluster in order to increase the energy efficiency of the protocol. This work extends the existing solutions by allowing multi-hop inter cluster communication in WSNs in which the direct communication between $\mathrm{CHs}$ or the sink is not possible due to the distance between them. Thus, the main innovation of the solution proposed here is that the multi-hop approach is followed inside the cluster and outside the cluster. $\mathrm{CHs}$ can also perform data fusion to the data receive, allowing a reduction in the total transmitted and forwarded data in the network [16]

\subsection{E-LEACH}

E-LEACH is based on LEACH protocol to balance the energy consumption of sensor nodes in order to solve the overload energy consumption problem. The E-LEACH adopts the same round concept with the original LEACH. In hierarchical routing protocols, the number of cluster-heads is a key factor that affects the performance of routing protocols. If the number of cluster-heads is less, each clusterhead needs to cover larger region, this will lead the problem that some cluster-members get far from their clusterheads and consume much more energy. As the communication between cluster heads and the base station needs much more energy than common nodes, the excessive number of cluster-heads will increase the energy consumption of the whole network and shorten the network lifetime. Therefore, it is necessary to select optimal cluster head number to make the energy consumption minimum. In the E-LEACH minimum spanning tree between cluster heads is used, choose the cluster head which has largest residual energy as the root node [17].

\subsection{LEACH-SM}

Is proposed in [18] where the spare selection phase is added to LEACH. The authors propose to add a spare selection phase between LEACH cluster setup and steady state phases. The spare nodes added into WSNs, are initially asleep but they are ready to be switched on when any active node uses up its energy. The problem is that when the spare node joins network, the whole network needs to be established again that consumes extra energy for other nodes.

\subsection{Multi-Hop LEACH}

It is difficult for all $\mathrm{CHs}$ to communicate directly with the base station when the sensors' deployed area is very large. High transmission power is required to send the data from $\mathrm{CHs}$ to the base station if the base station is far away from the CHs. LEACH only assumes that all $\mathrm{CHs}$ are at single hop distance from the base station, which is not a suitable approach for this scenario. An approach, Multi-Hop LEACH, addresses this issue [20]. Multi-Hop LEACH is an enhancement of LEACH, which reduces the energy consumption of the CHs in large WSNs [19,20, 21]. The formation of clusters and selection of the $\mathrm{CHs}$ is done in the setup phase. During steady state phase, the noncluster head nodes send their data to the $\mathrm{CHs}$. The $\mathrm{CHs}$ aggregate and transmit that data towards the base station directly or through other CHs. The two types of MultiHop LEACH communications are intra-cluster and intercluster.

The random selection of $\mathrm{CHs}$ is the same as in LEACH. The most feasible and energy efficient path is selected for that $\mathrm{CH}$ which is far from the base station. The method used for the selection of intermediate $\mathrm{CH}$ is the distance between the $\mathrm{CH}$ and the base station. The $\mathrm{CH}$ closer to the base station receives the data from the other $\mathrm{CH}$ which is far from base station. This helps to save energy of those $\mathrm{CHs}$ which belong to the clusters with larger distance from the base station as higher transmission energy cost is required for communication with larger distances. 


\subsection{Solar-aware LEACH (sLEACH)}

In some cases sensor nodes are deployed in remote or nonaccessible areas such as battlefields or jungles. One of the major issues in the sensor node is to maintain the required power. The life span of WSNs can be improved by using solar panels with sensor nodes. The solar-aware LEACH protocol is proposed for application specific WSNs to tackle these situations [22]. Some nodes are enhanced with solar power in sLEACH. The nodes with solar power are stronger candidates for the selection of $\mathrm{CHs}$. The concepts of LEACH and LEACH-C are used in SLEACH protocol.

* Problem Statement: Battery powered node die early because of high energy consumption and in big network it is very difficult to change battery of those node, as battle field, forest etc

* Solution: Energy conservation plays a crucial role in wireless sensor networks since such networks are designed to be placed in hostile and non-accessible areas While battery driven sensors will run out of battery sooner or later the use of renewable energy sources such as solar power or gravitation may extend the lifetime of a sensor network. We propose to utilize solar power in wireless sensor networks. Some nodes are enhanced with solar power in SLEACH. The nodes with solar power are stronger candidates for the selection of CHs. The concepts of LEACH and LEACH-C are used in SLEACH protocol.

* Limitation: As we know solar powered devices totally depend on solar energy if there were no sun then there will be a problem in network to work

\subsection{Solar-aware centralized LEACH}

In this protocol the base station uses the enhanced central control algorithm for the selection of the CHs. The nodes driven by a solar power system are given priority for the selection of $\mathrm{CHs}$ due to their higher residual energy. All nodes in sLEACH send their solar power status along with their ID, location, and energy information to the base station. sLEACH uses the same $\mathrm{CH}$ selection criteria as in LEACH-C (i.e. Nodes with higher energy will be chosen as CHs). The addition of solar-aware nodes helps to improve the performance of the entire network. The network performance and the life span of solar-aware nodes depend upon the duration of the node's exposure to the sun.

The shorter sun exposure reduces the node's residual energy which leads to a change in the role of the node from cluster head to non-cluster head [22]. On the other hand, a noncluster node can get more power than current $\mathrm{CH}$ with the help of a solar power system, which sends the message with the flag to $\mathrm{CH}$. This node will be selected as $\mathrm{CH}$.

* Problem Statement: Battery powered node die early because of high energy consumption and in big network it is very difficult to change battery of those node, such as battle field, forest etc

* Solution: In this protocol the base station uses the enhanced central control algorithm for the selection of the CHs. The nodes driven by a solar power system are given priority for the selection of $\mathrm{CHs}$ due to their higher residual energy.

* Limitation: The shorter sun exposure reduces the node's residual energy which leads to a change in the role of the node from cluster head to non-cluster head. On the other hand, a non-cluster node can get more power than current $\mathrm{CH}$ and they become $\mathrm{CH}$

\subsection{Solar-aware distributed LEACH}

The distributed fashion is used for the formation of clusters in solar-aware distributed LEACH [22]. It works in the same way as LEACH with two phases; setup phase and steady state phase.

(i) Setup Phase: The solar aware nodes are the stronger candidates during the selection process of the $\mathrm{CH}$. Initially the probability of a battery operated node is the same as a solar driven node. To give more priority, the probability of solar aware nodes needs to be higher than battery operated nodes. The formula given in equation 1 is changed by equation 2 to consider the solar aware nodes.

$$
T(n)=\frac{s f(n) * P}{1-(C H s / \text { numNodes })} \quad \text { equation }
$$

Where $s f(n)$ is the scaling factor.

The value of $s f(\mathrm{n})$ for solar driven node is greater than 1 .

The value of $s f(\mathrm{n})$ for battery driven node is the reciprocal of the solar driven node's $s f(\mathrm{n})$ value.

For example: If $s f(\mathrm{n})$ of the solar driven node is 4 then $s f(\mathrm{n})$ of the battery driven node will be $1 / 4$

$\mathrm{P}=$ Optimal $\mathrm{CH}$ percentage .

$\mathrm{CHs}=$ Number of cluster heads since the start

numNodes $=$ Total number of nodes .

The remaining process of the setup phase is the same as LEACH.

(ii) Steady state Phase: During steady state the member nodes send the data to the base station via $\mathrm{CH}$. The process of $\mathrm{CH}$ handover starts if the solar power level of a non-cluster head node increases more than the power level of the current $\mathrm{CH}$ node. The node with the higher energy level sends a flag to the current $\mathrm{CH}$ and is ultimately selected as the $\mathrm{CH}$ of the cluster.

The performance of distributed sLEACH increases by adding more solar operated nodes.

* Problem Statement: Battery powered node die early because of high energy consumption and in big network it is very difficult to change battery of those node such as battle field, forest etc

* Solution: The distributed fashion is used for the formation of clusters in solar-aware distributed LEACH . Two important condition

- Solar-powered nodes must become cluster heads with a higher probability.

- A node that has been solar-powered while being a cluster head should be able to become a cluster head again even during the next $1 / \mathrm{P}$ rounds.

* Limitation: The shorter sun exposure reduces the node's residual energy which leads to a change in the role of the node from cluster head to non-cluster head. On the other hand, a non-cluster node can get more power than current $\mathrm{CH}$ and they become $\mathrm{CH}$. 


\subsection{Selective Cluster LEACH (LEACH- $\mathrm{SC})$}

LEACH-SC protocol [23] introduces a new method of $\mathrm{CH}$ selection. Most of the previous methods use the residual energy and distance as parameters for the selection of $\mathrm{CH}$. A problem was observed in these methods of $\mathrm{CH}$ selection. The main task of $\mathrm{CH}$ is to route the member nodes data to the base station. Even when the member node is closer to the base station than its $\mathrm{CH}$, according to previous methods the node must send data to its $\mathrm{CH}$ first. The $\mathrm{CH}$ then transmits the same data to the base station. The energy cost increases in the case where the member nodes send the data to the $\mathrm{CH}$ in the opposite direction of the base station. The LEACH-SC mechanism resolves this issue, which results in lower energy cost of the sensor nodes and prolongs the network life. Like $\mathrm{LEACH}$, the LEACH-SC procedure works in rounds. Each round consists of setup and steady state phases. LEACH-SC improves the cluster formation portion of the setup phase. An enhanced cluster head formation algorithm is given in which only selected $\mathrm{CHs}$ transmit their IDs and location information to all nodes. The nodes receive this information based on their location. The information from the $\mathrm{CH}$ is received only by those nodes that are in that $\mathrm{CH}$ coverage range. The nodes choose only that $\mathrm{CH}$ which is closest to the base station and their own centre point. The selection of $\mathrm{CH}$ in this way reduces the overall energy consumption in steady state phase.

The mathematical analysis of LEACH-SC discussed in [23] is to prove the energy efficiency of the network when a sensor node selects a $\mathrm{CH}$ whose location lies nearest to the midpoint between the node itself and the base station.

\subsection{MODLEACH [24]}

Modified LEACH (MODLEACH) proposed by D. Mahmood, N. Javaid. They introduce efficient cluster head replacement scheme and dual transmitting power levels. IT is for homogeneous network. They use different power level for transmission from node to $\mathrm{CH}, \mathrm{CH}$ to $\mathrm{CH}, \mathrm{CH}$ to sink. They use $\mathrm{CH}$ selection method such that if $\mathrm{CH}$ has more energy than it remains as $\mathrm{CH}$ for next round. Also they introduce soft threshold and hard threshold; so energy consumption reduces.

They modify one of the most prominent wireless sensor network's routing protocols "LEACH" as modified LEACH (MODLEACH) by introducing efficient cluster head replacement scheme and dual transmitting power levels. Our modified LEACH, in comparison with LEACH out performs it using metrics of cluster head formation, throughput and network life. Afterwards, hard and soft thresholds are implemented on modified LEACH (MODLEACH) that boast the performance even more. Finally a brief performance analysis of LEACH, Modified LEACH (MODLEACH), MODLEACH with hard threshold (MODLEACHHT) and MODLEACH with soft threshold (MODLEACHST) is undertaken considering metrics of throughput, network life and cluster head replacements.

\subsubsection{Advantages of MODLEACH}

(i) It minimizing routing load of protocol and increase energy efficiency.

(ii) It distributes dissipation among Cluster Heads, Sensor Nodes.

\subsubsection{Limitations of MODLEACH}

(i) If cluster head has less energy than required threshold, it will be replaced according to LEACH algorithm. They used same equation same as LEACH rather than HEED/DEEC.

\subsection{Q-LEACH [25]}

In this work, Author proposes Quadrature-LEACH for homogenous a network which enhances stability period, network life-time and throughput quiet significantly.

According to this approach sensor nodes are deployed in the territory. In order to acquire better clustering we partition the network into four quadrants. Doing such sort of partitioning better coverage of the whole network is achieved. Additionally, exact distribution of nodes in field is also well defined.

\subsection{O-Leach [26]}

Optimize LEACH (O-LEACH) proposed by S. El Khediri and N. Nasri. In which Election of cluster-head in each round with energy value greater than ten percent of the residual value at each sensor. So node which has low energy not become $\mathrm{CH}$; so it consumes less energy and remain alive for next few round for communication.

\subsubsection{Advantages of O-LEACH}

(1) It achieves longer stability.

(2) It was improve energy efficiency as well as lifetime.

\subsubsection{Limitations of O-LEACH}

(1) If any nodes have not remaining energy greater than required then no $\mathrm{CH}$ selected network fails.

(2) They used same equation same as LEACH rather than HEED/DEEC.

\subsection{0 iMODLEACH [27]}

In this paper, we have proposed iMODLEACH protocol which is an extension to the MODLEACH protocol. Simulation results indicate that iMODLEACH outperforms MODLEACH in terms of network life-time and packets transferred to base station. The mathematical analysis helps to select particular wireless sensor network application. such values of these parameters which can suit a particular wireless sensor network application.

In this section we will discuss the iMODLEACH protocol in detail. This protocol is primarily based on MODLEACH [27] protocol which is itself an inspiration of the LEACH [3] protocol.

The type of network is heterogeneous. There are $\mathrm{n}$ numbers of nodes, randomly distributed across region. The main region is further divided into sub regions which are normally referred to as, clusters. Each cluster contains number of nodes of which one of them is acting as the $\mathrm{CH}$. Each $\mathrm{CH}$ receives data from all of its client nodes and performs some necessary iteration for compression. All CH's forward the compressed data to Base Station. All nodes are considered nomadic or stationary within their respective cluster and hence, there is no abrupt change in network topology.

\subsection{Z-LEACH [28]}

The proposed algorithm adds some features that LEACH does not consider such as:

1) Use the remaining energy of the present $\mathrm{CH}$ to select the next $\mathrm{CH}$. 
2) Use the least overloaded cluster head to add another level of aggregation.

The assumptions used to facilitate the design of the proposed clustering protocol are:

Assumption 1: All the sensor nodes are static and homogeneous in physical characteristics i.e. having identical sensing, computing and communicating ability. Initial battery powers of the nodes are identical at deployment.

Assumption 2: The current $\mathrm{CHs}$ will not drain out completely during next $\mathrm{CH}$ selection process.

Assumption 3: All nodes can transmit with enough power to reach the BS if needed. So the algorithm is not applicable to networks deployed in large region.

Assumption 4: Sensor nodes always have some data to send and nodes located close to each other have correlated data.

\subsection{TL-LEACH [29]}

Uses random rotation of local cluster base stations (primary cluster-heads and secondary cluster-heads). In this way we build, where it is possible, a two-level hierarchy. This permits to better distribute the energy load among the sensors in the network especially when the density of network is higher. TLLEACH uses localized coordination to enable scalability and robustness.

The TL-LEACH uses the following techniques to achieve energy and latency efficiency:

- Randomized, adaptive, self-configuring cluster formation.

- Localized control for data transfers.

\section{CONCLUSION}

All the version that mentioned above are progressed one step forward in the field of energy efficiency of LEACH protocol, but there are more ways that needs to be explore and to be implemented in the future. With the help of these protocols more version are yet to be modified.

\section{REFERENCES}

[1] Ming Yu, Leung, K.K. And Malvankar, "A dynamic clustering and energy efficient routing technique for sensor networks", Published in: IEEE on Wireless Communications, Date: August 2007, Vol: 6(8), Page(s): 3069-3079

[2] F. Bouabdallah, N. Bouabdallah and R. Boutaba. "CrossLayer Design for Energy Conservation in Wireless Sensor Networks", Published in: IEEE International Conference on Communications, Date: June 2009, Accession Number: 10815184

[3] Wendi Rabiner Heinzelman et al."Energy-Efficient Communication Protocol for Wireless Micro sensor Networks", Published in: In Proceeding of the 33rd Hawaii International Conference on System Sciences, Date of Conference: 4-7 Jan. 2000, Page(s): 1-10

[4] W. B. Heinzelman. "Application-Specific Protocol Architectures for Wireless Networks", PhD thesis, Massachusetts Institute of Technology, June 2000 Boston.

[5] Younis, O. Fahmy, Sonia. "HEED: A hybrid, energyefficient, distributed clustering approach for ad-hoc sensor networks", Published in: Mobile Computing, IEEE Transactions on (Volume:3, Issue: 4 ) Date of Publication: Oct.-Dec. 2004, Page(s): 366 - 379
[6] Arati Manjeshwar, Agrawal, D.P.. "TEEN: A protocol for enhanced efficiency in wireless sensor networks", Published in: Parallel and Distributed Processing Symposium, Proceedings 15th International Conference, Conference Location: San Francisco, CA, USA, vol. 3, Date of Conference: 23-27 April 2000 Page(s): 2009 2015

[7] Md. Atiqur Rahman, Shahed Anwar, Md. Ileas Pramanik, Md. Ferdous Rahman, "A Survey on Energy Efficient Routing Techniques in Wireless Sensor Network", Published in Advanced Communication Technology (ICACT), 2013 15th International Conference, Date of Conference: 27-30 Jan. 2013 Conference Location : PyeongChang, Page(s): 200 - 205

[8] J.Gnanambigai, Dr.N.Rengarajan, K.Anbukkarasi, "Leach and Its Descendant Protocols: A Survey", Published in: International Journal of Communication and Computer Technologies Volume 01 - No.3, Issue: 02, Date: September 2012, Page(s): 15-21

[9] Ezzati abdellah, Said benalla, Abderrahim Beni hssane, Moulay Lahcen Hasnaoui, "Advanced Low Energy Adaptive Clustering Hierarchy", Published in: (IJCSE) International Journal on Computer Science and Engineering, Volume-02 Issue-07, 2010, Page(s): 24912497.

[10] Zahoor A. Khan and Srinivas Sampalli, "AZRLEACH: An Energy Efficient Routing Protocol for Wireless Sensor Networks", Published in: International Journal of Communications, Network \& System Sciences, , Vol. 5 No. 11, 2012, Page(s): 785-795.

[11] C. Intanagonwiwat, R. Govindan, D. Estrin, "Directed Diffusion: A scalable and robust communication paradigm for sensor networks", Published in: Proceedings of the 6th Annual ACM/IEEE International Conference on Mobile Computing and Networking (MobiCom00), Boston, MA, August 2000.

[12] P. Manimala1, R. Senthamil selvi2, "A Survey on Leach-Energy Based Routing Protocol", Published in: International Journal of Emerging Technology and Advanced Engineering, Website: www.ijetae.com (ISSN 2250-2459, ISO 9001:2008 Certified Journal, Volume - 3, Issue-12, December 2013).

[13] Mu Tong, Minghao Tang, "LEACH-B: An Improved LEACH Protocol for Wireless Sensor Network", Published in: Wireless Communications Networking and Mobile Computing (WiCOM), 2010 6th International Conference on, Chengdu, , E-ISBN: 978-1-4244-3709-2, Print ISBN: 978-1-4244-3708-5, Publisher: IEEE, Date: 23-25 Sept. 2010. Page(s): $1-4$

[14] Parul Bakaraniya , Sheetal Mehta, "K-LEACH: An improved LEACH Protocol for Lifetime Improvement in WSN" Published in: International Journal of Engineering Trends and Technology (IJETT) - Volume 4 Issue - 5, May 2013, Page(s): 1521- 1526

[15] M. Bani Yassein, A. Al-zou'bi, Y. Khamayseh, W. Mardini, "VLEACH: Improvement on LEACH Protocol of Wireless Sensor Network", Published in: International Journal of Digital Content Technology and its Applications, Volume - 3, Issue - 2, June 2009 Page(s): $132-136$

[16] Hwa Young Lim, Sung Soo Kim, Hyun Jun Yeo, Seung Woon Kim, and Kwang Seon Ahn "Maximum Energy Routing Protocol based on Strong Head in Wireless Sensor Networks" Published in: Sixth International Conference on Advanced Language Processing and Web Information Technology, Location: Luoyang, Henan, 
China, Publisher: IEEE, Date of Conference: 22-24 Aug. 2007, Page(s): $414-419$

[17] Jia Xu,Ning Jin, Xizhong Lou,Ting Peng,Qian Zhou, Yanmin Chen, "Improvement of LEACH protocol for WSN", Published in: 9th International Conference on Fuzzy Systems and Knowledge Discovery (FSKD), Date of Conference: 29-31 May 2012, Location: Sichuan. Publisher: IEEE, Page(s): 2174 - 2177

[18] Bakr, Lilien L, "LEACH-SM: A protocol for extending wireless sensor network lifetime by management of spare nodes", Published in: Innovative Mobile and Internet Services in Ubiquitous Computing (IMIS), 2011 Fifth International Conference on, Date of Conference: June 30 2011-July 2 2011, Location: Seoul, Publisher: IEEE, Page(s): 277 - 282

[19] Fan Xiangning and Song Yulin, "Improvement on LEACH Protocol of Wireless Sensor Network," Published in: SENSORCOMM '07 Proceedings of the 2007 International Conference on Sensor Technologies and Applications, IEEE Computer Society Washington, DC, USA (92007, Pages: 260-264

[20] R. V. Biradar, D. S. R. Sawant, D. R. R. Mudholkar and D. V. C. Patil, "Multi- hop Routing In Self-Organizing Wireless Sensor Networks", Published in: IJCSI International Journal of Computer Science, vol. 8, no. 1, 2011.

[21] N. Israr and I. Awan, "Multihop clustering Algorithm for load balancing in Wireless Sensor Networks", Published in: International Journal of Simulation, Systems, Science and Technology, vol. 8, no. 1, Pages: 13-25, 2007.

[22] T. Voigt, H. Ritter, J. Schiller, A. Dunkels and J. Alonso, "Solar-aware Clustering in Wireless Sensor Networks," Published in: Proceedings of the Ninth IEEE Symposium on Computers and Communications, 2004.

[23] W. Jun, Z. Xin, X. Junyuan and M. Zhengkun, "A Distance-based Clustering Routing Protocol in Wireless Sensor Networks," Published in: Important national science technology specific projects, 2011.
[24] D. Mahmood, N. Javaid, S. Mehmood, S. Qureshi, A.M. Memon, T. Zaman, "MODLEACH: a variant of

LEACH for WSNs", Published in: 26th IEEE Canadian Conference on Electrical and Computer Engineering (CCECE2013), Regina, Saskatchewan, Canada, 2013

[25] B. Manzoor, N. Javaid, O. Rehman, M. Akbar, Q. Nadeem, A. Iqbal, M. Ishfaq, "Q-LEACH: A New Routing Protocol for WSNs", Published in: International Workshop on Body Area Sensor Networks (BASNet2013) in conjunction with 4th International Conference on Ambient Systems, Networks and Technologies (ANT 2013), 2013, Halifax, Nova Scotia, Canada, Procedia Computer Science, Volume 19, 2013, Pages: 926-931, ISSN 1877-0509.

[26] S. El Khediri, N. Nasri, A. Wei, A. Kachourid, "A New Approach for Clustering in Wireless Sensors Networks Based on LEACH", Published by: ELSEVIER, Procedia Computer Science 32 Pages: 1180 - 1185, 2014

[27] S.Ahmed, M. M. Sandhu, N. Amjad, A. Haider, M. Akbar, A. Ahmad, Z. A. Khan, U. Qasim, N. Javaid, "iMOD LEACH: improved MODified LEACH Protocol for Wireless Sensor Networks", Published in: Journal of Basic and Applied Scientific Research, arXiv preprint arXiv:1309.4379, Publication date: 17 Sep. 2013

[28] Subhadra Shaw (Bose), "Z-LEACH: Energy-Efficient Routing Protocol in Wireless Sensor Network", In Published in: International Journal of Scientific \& Engineering Research, ISSN 2229-5518, Volume - 2, Issue - 12, December-2011,

[29] V. Loscrì, G. Morabito, S. Marano, "TL-LEACH: A Two-Levels Hierarchy for Low-Energy Adaptive Clustering Hierarchy", Published in: Vehicular Technology Conference, 2005, IEEE 62nd Volume-3, Date of Conference: 25-28 Sept 2005, Page(s): 1809 1813 